
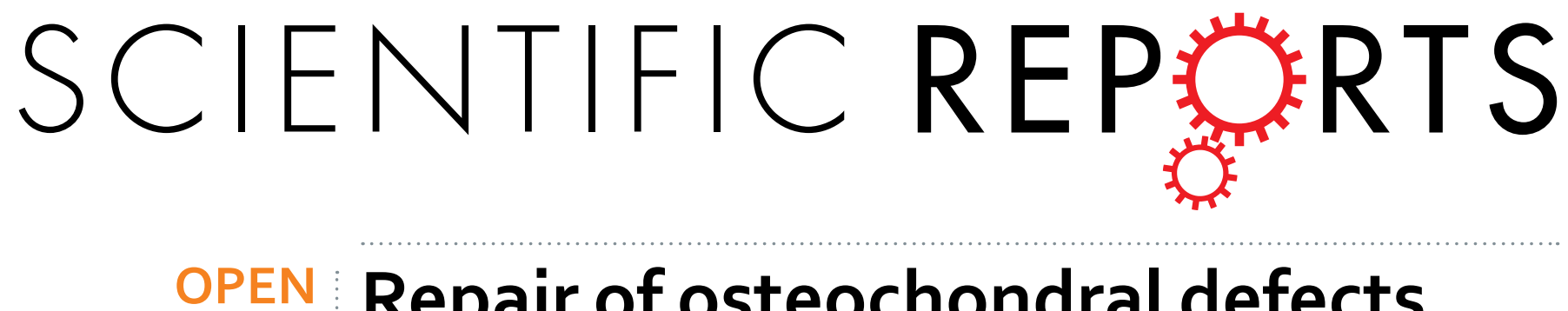

\title{
Repair of osteochondral defects
} with in vitro engineered cartilage based on autologous bone marrow

Received: 06 September 2016

Accepted: 06 December 2016

Published: 13 January 2017

\section{stromal cells in a swine model}

\author{
Aijuan He ${ }^{1,2, *}$, Lina Liu ${ }^{1,2, *}$, Xusong Luo ${ }^{1}$, Yu Liu ${ }^{1,2}$, Yi Liu $^{1,2}$, Fangjun Liu ${ }^{3}$, Xiaoyun Wang ${ }^{4}$, \\ Zhiyong Zhang ${ }^{1,2}$, Wenjie Zhang ${ }^{1,2}$, Wei Liu ${ }^{1,2}$, Yilin Cao ${ }^{1,2}$ \& Guangdong Zhou ${ }^{1,2,3}$
}

Functional reconstruction of large osteochondral defects is always a major challenge in articular surgery. Some studies have reported the feasibility of repairing articular osteochondral defects using bone marrow stromal cells (BMSCs) and biodegradable scaffolds. However, no significant breakthroughs have been achieved in clinical translation due to the instability of in vivo cartilage regeneration based on direct cell-scaffold construct implantation. To overcome the disadvantages of direct cell-scaffold construct implantation, the current study proposed an in vitro cartilage regeneration strategy, providing relatively mature cartilage-like tissue with superior mechanical properties. Our strategy involved in vitro cartilage engineering, repair of osteochondral defects, and evaluation of in vivo repair efficacy. The results demonstrated that BMSC engineered cartilage in vitro (BEC-vitro) presented a time-depended maturation process. The implantation of BEC-vitro alone could successfully realize tissue-specific repair of osteochondral defects with both cartilage and subchondral bone.

Furthermore, the maturity level of BEC-vitro had significant influence on the repaired results. These results indicated that in vitro cartilage regeneration using BMSCs is a promising strategy for functional reconstruction of osteochondral defect, thus promoting the clinical translation of cartilage regeneration techniques incorporating BMSCs.

Functional repair of large osteochondral defects is always a great challenge in orthopaedic surgery because of complex osteochondral structure and the limited regeneration ability of cartilage ${ }^{1}$. Tissue engineering, which can regenerate live and functional tissue similar to native tissue, may provide a promising strategy ${ }^{2,3}$. In fact, autologous chondrocyte implantation/transplantation (ACI/ACT) has been approved by the United States Food and Drug Administration for clinical treatment of articular cartilage defects ${ }^{4-6}$. However, strategies using chondrocytes as a cell source will inevitably be associated with limited cell supply, donor site morbidity, and most importantly, restoration of only the cartilage layer but not underlying subchondral bone $e^{7-9}$. Therefore, identification of a more appropriate cell source to promote regeneration of both cartilage and subchondral bone is an urgent issue.

Bone marrow stromal cells (BMSCs) are considered an ideal cell source for osteochondral regeneration because of insignificant donor-site morbidity, robust proliferative capacity, and committed potentials for both cartilage and bone $\mathrm{e}^{10-12}$. Many studies, including our previous investigations, have already demonstrated that BMSCs could repair osteochondral defects with both regenerated cartilage and bone under the regulation of articular osteochondral microenvironments ${ }^{1,13,14}$. However, most of these reports were based on cell-scaffold constructs as implants, which have several disadvantages for both surgical manipulation and tissue regeneration, such as inconvenient handling for surgeons, cell leakage ${ }^{15}$, inflammatory reaction triggered by abundant un-degraded scaffolds ${ }^{16,17}$, difficulty in quality control prior to implantation, insufficient mechanical properties, and spontaneous differentiation within the traumatic environment after implantation ${ }^{18,19}$. Therefore, the total

${ }^{1}$ Department of Plastic and Reconstructive Surgery, Shanghai 9th People's Hospital, Shanghai Jiao Tong University School of Medicine, Shanghai Key Laboratory of Tissue Engineering, Shanghai, P.R. China. ${ }^{2}$ National Tissue Engineering Center of China, Shanghai, P.R. China. ${ }^{3}$ Research Institute of Plastic Surgery, Wei Fang Medical College, Wei Fang, Shandong, China. ${ }^{4}$ Department of General Surgery, Wu Jing Hospital, Minhang District, Shanghai, China. ${ }^{*}$ These authors contributed equally to this work. Correspondence and requests for materials should be addressed to X.W. (email: gaokongliuyun@126.com) orY.C. (email: yilincao@yahoo.com) or G.Z. (email: guangdongzhou@126.com) 

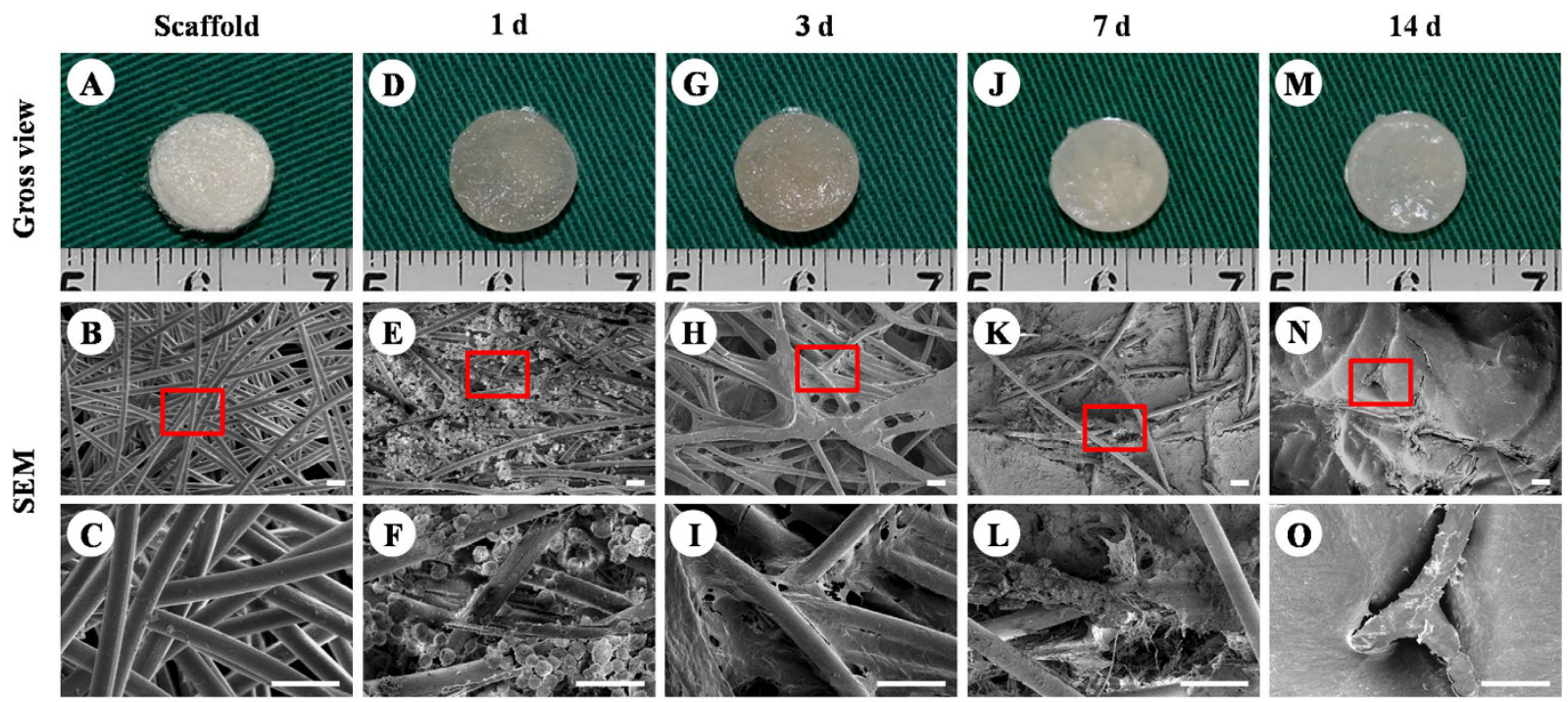

Figure 1. ECM production by BMSCs on PGA/PLA scaffolds. Unwoven PGA fibres were compressed to form a cylindrical-shaped scaffold with porous structure (A-C). BMSCs on the scaffold display round shapes within $24 \mathrm{~h}$ and distribute throughout PGA fibres (D-F). Three days after cell seeding, BMSCs started to spread and connect to PGA/PLA fibres with little ECM production $(\mathbf{G}-\mathbf{I})$. At 7 days, BMSCs produced enough ECM to wrap around PGA fibres and cover the porous structure (J-L). At 2 weeks, constructs presented an ivory-white appearance and the PGA fibres had been completely covered by abundant ECM (M-O). Scale bar $=50 \mu \mathrm{m}$.

success rate of osteochondral defect repair is not satisfactor $y^{20}$, which obviously limits further clinical translation of cell-scaffold constructs.

We propose that in vitro cartilage regeneration is the key to solve these problems. There are many advantages of in vitro cartilage regeneration compared with in vivo chondrogenesis ${ }^{21}$, especially for stem cell-based cartilage regeneration. Primary advantages include convenient handling for surgeons (similar to autologous cartilage transplantation), reduced cell leakage ${ }^{15}$, mild inflammatory reaction because of minimal or no remnant scaffold $^{16,17}$, convenient quality control before implantation, superior mechanical properties, and more reliable cartilage regeneration after implantation (cartilage had formed before implantation and, thus, was less influenced by the traumatic environment) ${ }^{18,19}$. Despite these advantages, some important issues are still unknown. First, what is the cartilage formation process for BMSC in vitro engineered cartilage (BEC-vitro)? Second, can implantation of BEC-vitro alone realize tissue-specific repair of articular osteochondral defects with both cartilage and subchondral bone in a large animal model? Third, does the maturity level of BEC-vitro affect the efficacy of repair? And finally, whether prolonged in vitro pre-culture has influence on in situ integration of the implant? All these issues directly restrict the clinical translation of BEC-vitro and, thus, require thorough investigation.

To address these issues in this study, hybrid pigs, whose knee joints were very close to human's ones in structure and load condition, were employed as an animal model. Autologous BMSCs were seeded into polyglycolic acid/polylactic acid (PGA/PLA) scaffolds, and were chondrogenically induced for 2-12 weeks. The cartilage formation process and the hypertrophic character of BEC-vitro were investigated at different time points. Based on this, in vitro engineered constructs at 2, 4, and 8 weeks were used to repair autologous articular osteochondral defects, in order to clarify the feasibility, superiority, and optimal implantation timeline of BEC-vitro for repairing articular osteochondral defects. The current study provides detailed insights for future clinical applications of in vitro engineered cartilage incorporating BMSCs.

\section{Results}

Extracellular matrix (ECM) production by BMSCs on PGA/PLA scaffolds. ECM production by BMSCs on PGA/PLA scaffold was first evaluated by scanning electron microscopy (SEM) during the early stage of in vitro culture. PGA/PLA scaffolds maintained a cylindrical shape with porous structures (Fig. 1A-C). After cell seeding, the constructs still maintained their original shape and size, and gradually presented an ivory-white appearance with the culture time (Fig. 1D,G,J,M). SEM showed BMSCs on PGA fibres exhibited round shapes within 24 hours (Fig. 1E,F), and then, with increased ECM production, gradually spread to wrap around the PGA fibres as in vitro culture time progressed (Fig. 1H,I,K,L,N,O). Collectively, these results indicated that BMSCs maintained good ECM production ability on PGA/PLA scaffolds.

Gross and histological evaluation of BEC-vitro. The quality of in vitro cartilage regeneration and its potential of endochondral ossification are key factors influencing the efficacy of osteochondral defect repair ${ }^{22}$. Therefore, gross and histological evaluations of BEC-vitro were first performed to investigate in vitro cartilage formation and its hypertrophic character. With increased in vitro induction time, the constructs gradually presented a cartilaginous ivory-white appearance (Fig. 2). Histological examination showed that, with increased in vitro culture time, constructs gradually displayed mature cartilage features with typical lacuna structures, increased ECM deposition, and strongly positive staining of cartilage-specific matrices, such as sulfated glycosaminoglycan 


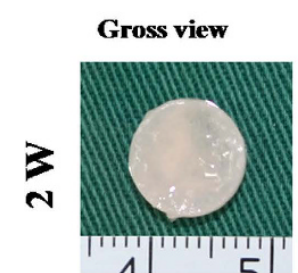

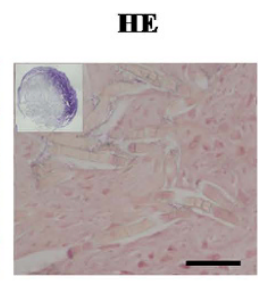

SAF-O
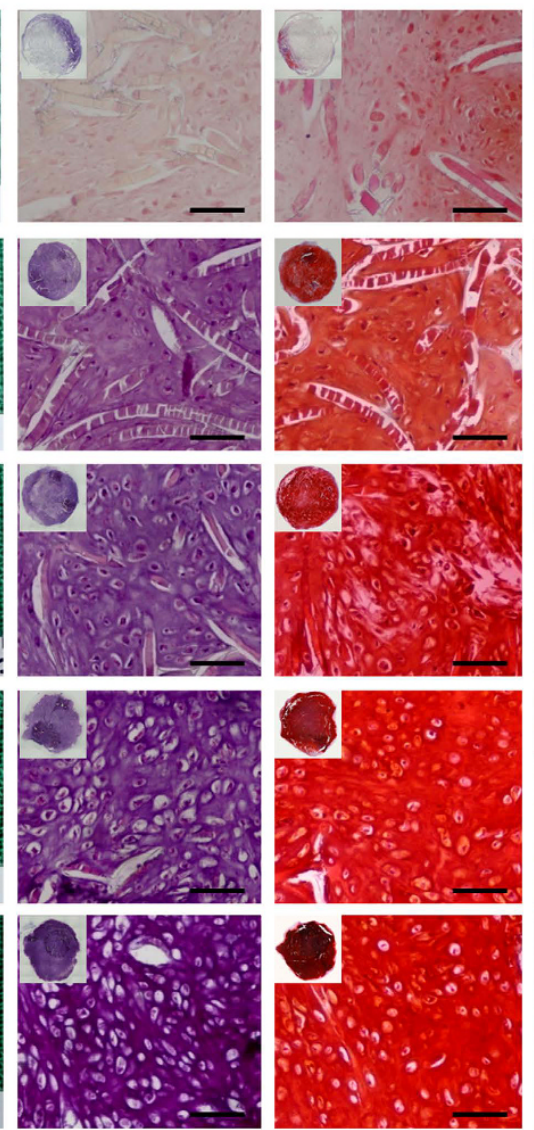

COL II

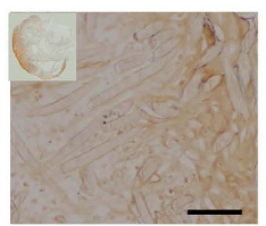

COL I
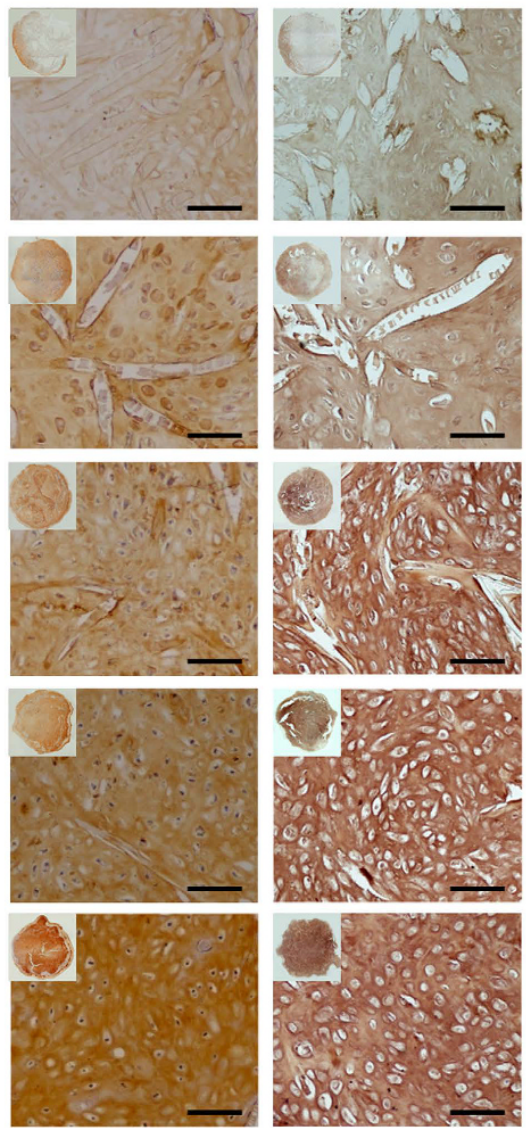
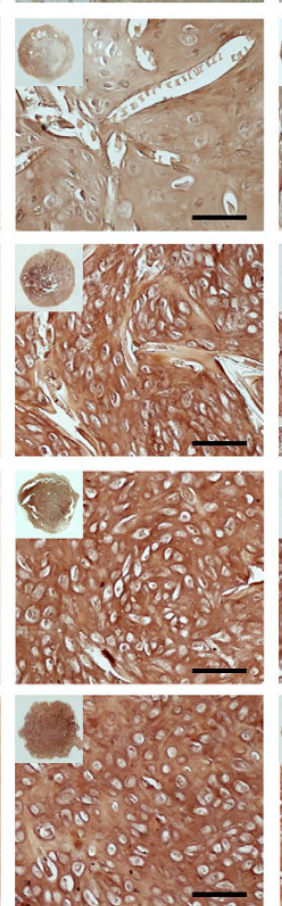

$\operatorname{COL} \mathbf{x}$
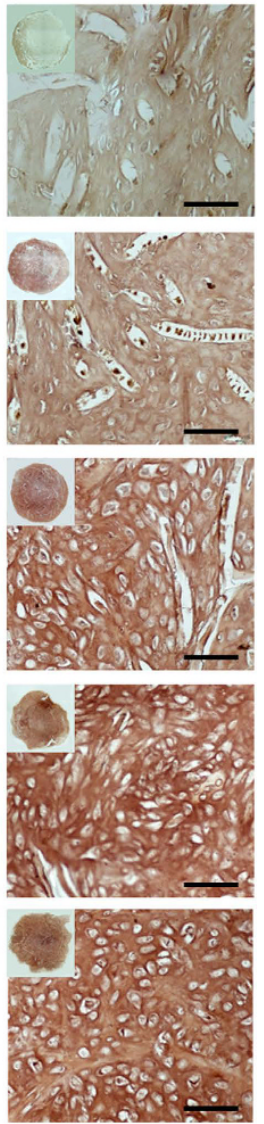

Figure 2. Gross view and histology of in vitro BMSC cartilage formation. In vitro chondrogenesis and maturation of BMSCs represented a time-dependent manner. At 2 weeks, constructs started to form cartilagelike tissue at the edge with ECM deposition; at 4 weeks, the constructs basically formed homogeneous, cartilagelike tissue with uniform ECM deposition and un-degraded PGA fibres; at 6 weeks, newly formed cartilage became more mature with typical lacuna structures and less residual PGA; at 8 and 10 weeks, samples became mature, homogeneous cartilage-like tissue with abundant lacuna structures and strongly positive staining for cartilage-specific matrices. Notably, expression of COL I and COL X was detected in all samples at different time points. Scale bar $=100 \mu \mathrm{m}$.

(GAG) and collagen type II (COL II; Fig. 2). Generally, preliminarily cartilage formation occurred at 4 weeks and achieved a mature and homogeneous state by 8 weeks, indicating a time-dependent trend of in vitro chondrogenesis and maturation (Fig. 2).

It was worth noticing that expression of the hypertrophy-related proteins such as collagen type I and $\mathrm{X}$ (COL I, COL X) was detected in all BEC-vitro samples at different time points, indicating that BEC-vitro maintained endochondral ossification potential even under a chondrogenic culture system (Fig. 2). In addition, residual PGA fibres showed a decreased trend for both number and length with increased in vitro culture time (Supplementary Fig. 1).

Biomechanical and biochemical evaluations of BEC-vitro. Biomechanical and biochemical evaluations of BEC-vitro further confirmed the above observations (Fig. 3). PGA/PLA scaffolds alone and BEC-vitro constructs at 2-week group showed poor mechanical strength (Supplementary Videos 1-2; Fig. 3F). BEC-vitro exhibited increased mechanical strength with good elasticity at 4 weeks, and achieved much better mechanical properties at 8 weeks (Supplementary Videos 3-4; Fig. 3F). All quantitative examinations related to cartilage maturity level, such as wet weight, contents of cartilage ECM (total GAG, total collagen, and COL II), and Young's modulus, significantly increased with in vitro induction time (Fig. 3A-D,F; $p<0.05$ ). However, COL I content didn't showed significant increased during in vitro culture (Fig. $3 \mathrm{E} ; \mathrm{p}>0.05$ ). These results indicated that in vitro chondrogenic induction could significantly promote BMSCcartilage formation with increased culture time.

Quantitative Real-Time polymerase chain reaction (QRT-PCR) analysis of BEC-vitro. Cartilage specific genes and hypertrophy-related genes were further analysed by qRT-PCR to evaluate the in vitro cartilage formation and its endochondral ossification potential at various time points. According to the current results, expression levels of cartilage specific genes COL IIA1, aggrecan (ACAN), and Sry related HMG box-9 (SOX 9) (Fig. 3G-I) rapidly increased with in vitro culture time and even achieved a much higher level than those found 
A

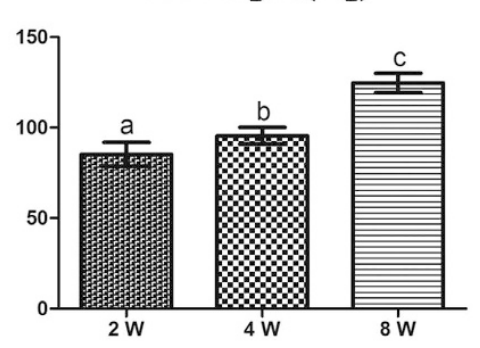

D

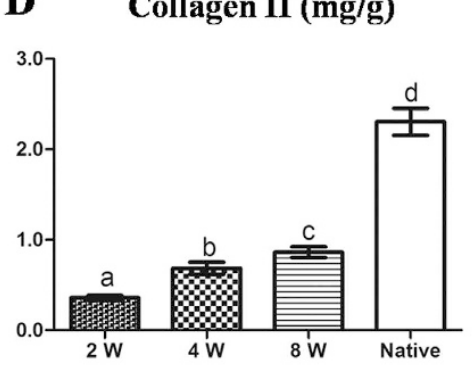

G

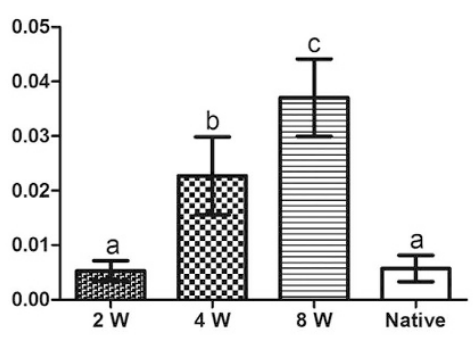

J COLIA1 $(2-\triangle C T)$

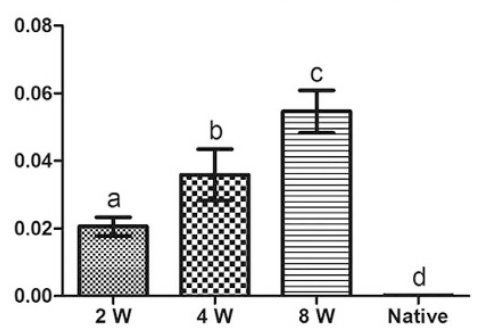

B

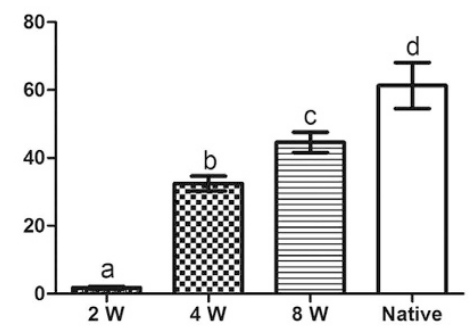

E Collagen I (mg/g)

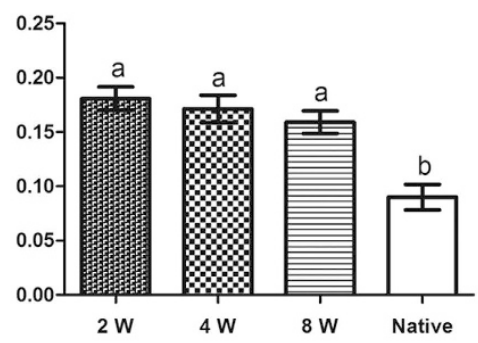

H ACAN (2- $\triangle \mathrm{CT})$

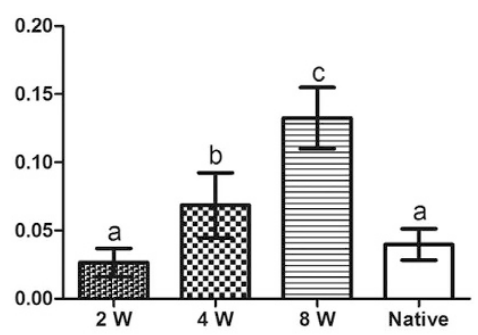

K COLXA1 $(2-\triangle \mathrm{CT})$

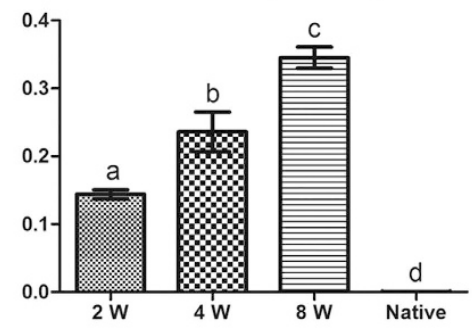

C Total collagen (mg/g)

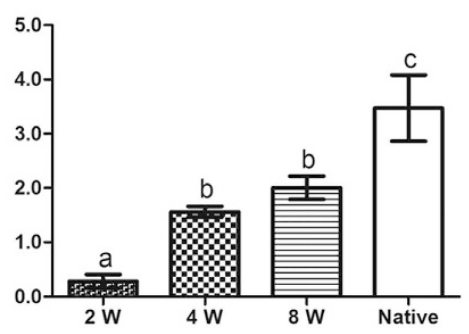

F Young's modulus (MPa)

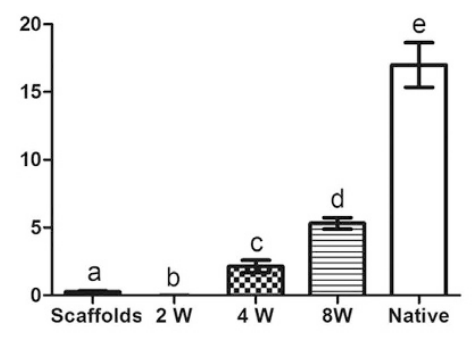

I $\quad \operatorname{sox} 9\left(2^{-\triangle C T}\right)$

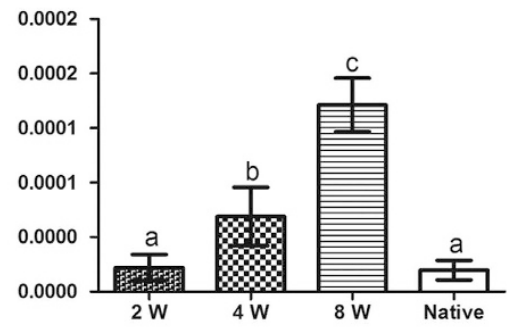

L MMP13 (2- $\triangle \mathrm{CT})$

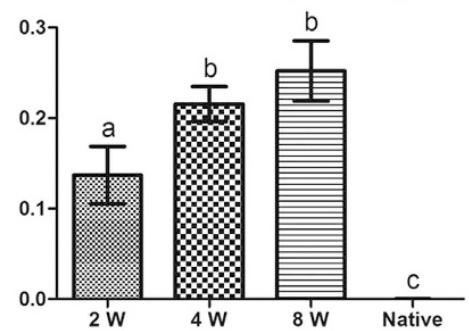

Figure 3. Biochemical, biomechanical and cartilage-related gene analyses of in vitro engineered tissues at various time points. All examinations of wet weight (A), GAG (B) contents, total collagen (C), total collagen II (D), Young's modulus (F), expressions of cartilage-related genes (G-I), and hypertrophy-related genes

(J-L), except for collagen I content (E), showed an increasing trend with in vitro induction time. Expression of cartilage-related genes in samples at 4 and 8 weeks were even higher than those found in native cartilage. The columns with different letters indicate statistical significance.

in native articular cartilage after 4 weeks $(\mathrm{p}<0.05)$. Surprisingly, hypertrophy-related genes COL IA1, COL XA1, and matrix metal proteinase 13 (MMP13) also increased with in vitro culture time (Fig. 3J-L). These results indicated that in vitro chondrogenic induction not only promoted chondrogenic differentiation of BMSCs but also activated their endochondral ossification potential.

Gross view and grading of repaired regions. As shown in Fig. 4, most defects in the 2-week group were repaired by fibrous-like tissue deep into subchondral bone areas (Fig. 4A,C). In contrast, more than half of the defects in the 4- and 8-week groups were completely repaired by cartilage- and bone-like tissues (Fig. 4D-F,G; Supplementary Fig. 2). These results indicate prolonged in vitro chondrogenic induction of BMSCs may improve 

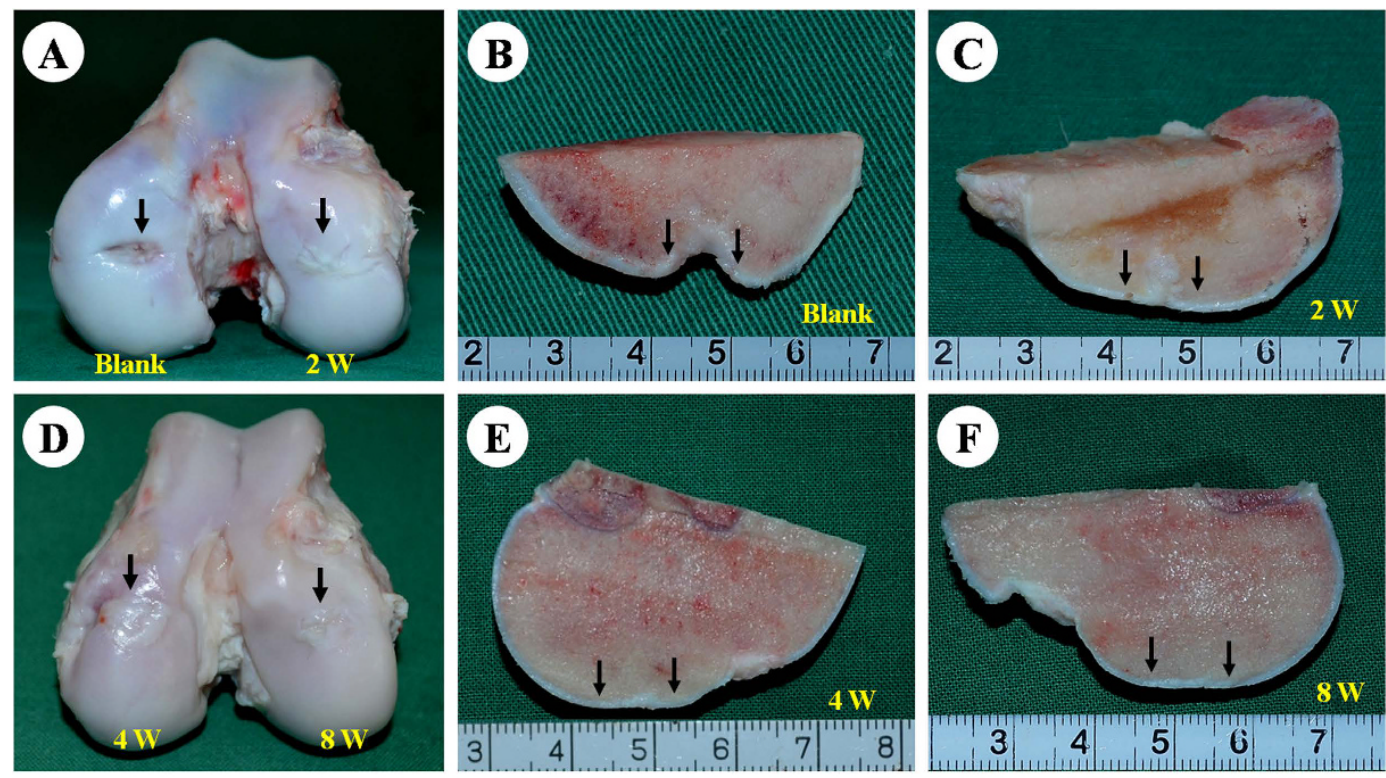

\section{G: Gross view grading table}

\begin{tabular}{lcccc} 
Grading & 2-week group & 4-week group & 8-week group & blank \\
\hline Complete repair & $1 / 9$ & $10 / 18$ & $11 / 19$ & $0 / 6$ \\
Incomplete repair & $6 / 9$ & $5 / 18$ & $5 / 19$ & $0 / 6$ \\
No repair & $2 / 9$ & $3 / 18$ & $3 / 19$ & $6 / 6$ \\
\hline
\end{tabular}

Figure 4. Gross view and grading of repaired regions. Most osteochondral defects in the 2-week group and all of the defects in the blank group were covered by fibrous-like tissue that infiltrated deep into subchondral bone; irregular surfaces were also observed (A-C). Defects in the 4- and 8-week groups were primarily repaired by glistening, white cartilage-like tissue and bone-like tissue exhibiting relatively smooth surfaces, good integration, and cartilage thickness similar to native structures (D-F). The detailed gross-view grading was listed in the grading table $(\mathbf{G})$. Black arrows indicate repaired regions.

the efficacy of osteochondral defect repair. Noticeably, in contrast to 4 - and 8-week groups, all defects in the blank group left obvious tissue defect or were mainly filled with fibrous tissues (Fig. 4A,B,G), implying the implanted BEC-vitro might directly participate in both articular cartilage and subchondral bone regeneration. Nevertheless, several defects in the 4 - and 8-week groups still showed no repair, which may result from individual difference or some unclear negative factors (Fig. 4G).

Histological examination of repaired regions. Histological examinations further supported the above observations. Repaired regions in the 2-week and blank groups mainly consisted of fibrous tissue that was negative for GAG, COL II, and osteocalcin [(OCN), (Figs 5, 6 and 7)], and strongly positive for COL I (Supplementary Fig. 3). In contrast, repaired regions in the 4- and 8-week groups were repaired primarily by cartilage-like tissue and cancellous bone, with strong cartilage ECM staining in cartilage-repaired regions and strong bone-marker expression in bone-repaired regions, confirming the tissue-specific repair of osteochondral defects in these two groups (Figs 5, 6 and 7; Supplementary Figs 2-3). In addition, histological grading scores further demonstrated that the 4- and 8-week groups achieved much better repair than the 2-week and blank groups (Supplementary Table 1). Collectively, these results support the notion that sufficient in vitro chondrogenic induction of BMSCs was necessary to improve the efficacy of osteochondral defect repair. Nevertheless, interfaces between repaired and native cartilage in the 4- and 8-week groups could still be distinguished by differences in cartilage thickness, surface regularity, and cell density (Figs 5, 6 and 7; Supplementary Fig. 2). These results further implied that the implanted BEC-vitro might directly participate in cartilage regeneration. Furthermore, neocartilage in the central area of defects basically presented negative expression of collagen I, implying these newly formed cartilage primarily consisted of hyaline-like cartilage (Supplementary Fig. 3). 

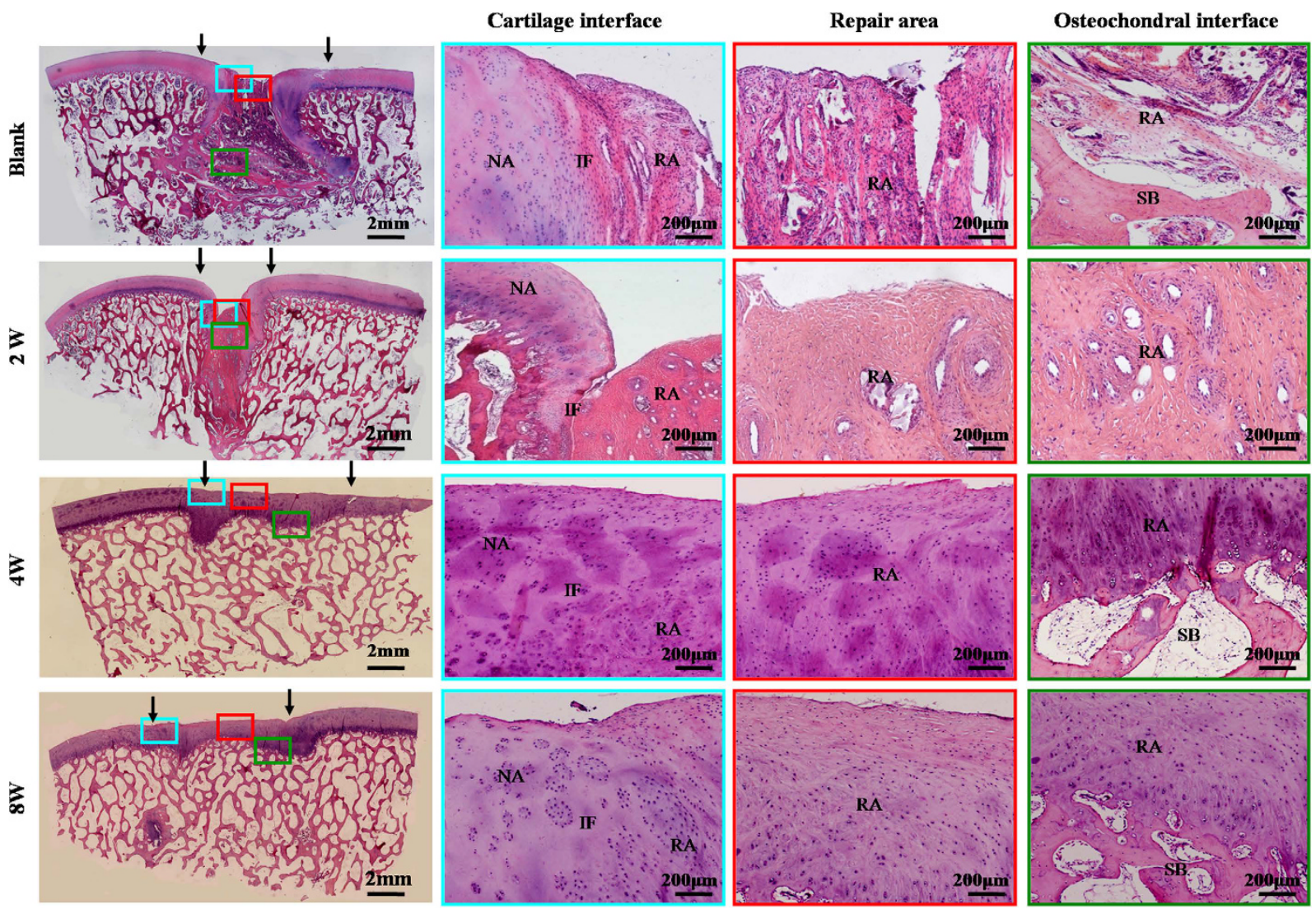

Figure 5. HE examination of repaired regions. Repaired tissues in the 2-week and blank groups showed signs of fibrogenesis and partial ossification with clear interface to the native tissue. Repaired tissues in the 4- and 8 -week groups were primarily composed of cartilage- and bone-like tissues. Newly formed cartilage in these two groups exhibited a higher cell density than the surrounding native cartilage, with mature lacuna structures and satisfactory interface integration of both cartilage and osteochondral interfaces. NA: native area; IF: interface; RA: repaired area; SB: subchondral bone. Black arrows indicate repaired regions.

Biomechanical and biochemical evaluations of the repaired tissues. In general, neocartilage in the 4- and 8-week groups showed higher cartilage ECM contents (GAG, total collagen, and collagen II) and stronger mechanical properties compared with the 2 -week and blank groups (Fig. 8A-C,E,F; $p<0.05$ ). Whereas, collagen I contents of neocartilage presented a contradictory trend amongst these groups, which was consistent with the histological examination (Fig. 8D; Supplementary Fig. 3). Noticeably, no significant differences were observed between the 4- and 8-week groups in any of the examinations described above, and all the examinations in these two groups were close to native cartilage (Fig. 8A-F). These results indicated repaired cartilage in the 4- and 8-week groups achieved levels similar to native articular cartilage with regard to both cartilage quality and function.

\section{Discussion}

Although some studies have reported the feasibility of repairing articular osteochondral defects using BMSC-scaffold constructs ${ }^{13,23}$, no significant breakthroughs have been achieved in stable reparative outcomes because multiple disadvantages of cell-scaffolds have apparently impeded the clinical translation of this approach $^{1,17,24}$. In vitro cartilage regeneration strategies may help to address this issue. The current study revealed that BMSC in vitro engineered cartilage presented a time-depended maturation process and confirmed that the implantation of BEC-vitro alone could successfully realize tissue-specific repair of osteochondral defects with both cartilage and subchondral bone. Furthermore, the maturity level of BEC-vitro had significant influence on the repaired results. These results suggest that BMSC in vitro engineered cartilage represents a promising strategy for clinical repair and recovery of osteochondral defects.

In vitro cartilage regeneration is the key point we proposed to avoid the disadvantages of direct cell-scaffold construct implantation. The current results showed that in vitro cartilage formation by BMSCs presented a gradually mature course with increased induction time and was accompanied by gradual degradation of scaffolds, which assisted in avoiding cell leakage ${ }^{15}$, inflammatory reactions triggered by scaffolds ${ }^{16,17}$, and spontaneous differentiation of BMSCs after implantation ${ }^{18,19}$. Furthermore, BEC-vitro achieved appropriate mechanical strength for surgical operation and was convenient for quality control measures before in vivo implantation, which facilitates more stable cartilage regeneration in vivo compared with cell-scaffold constructs. More importantly, high 

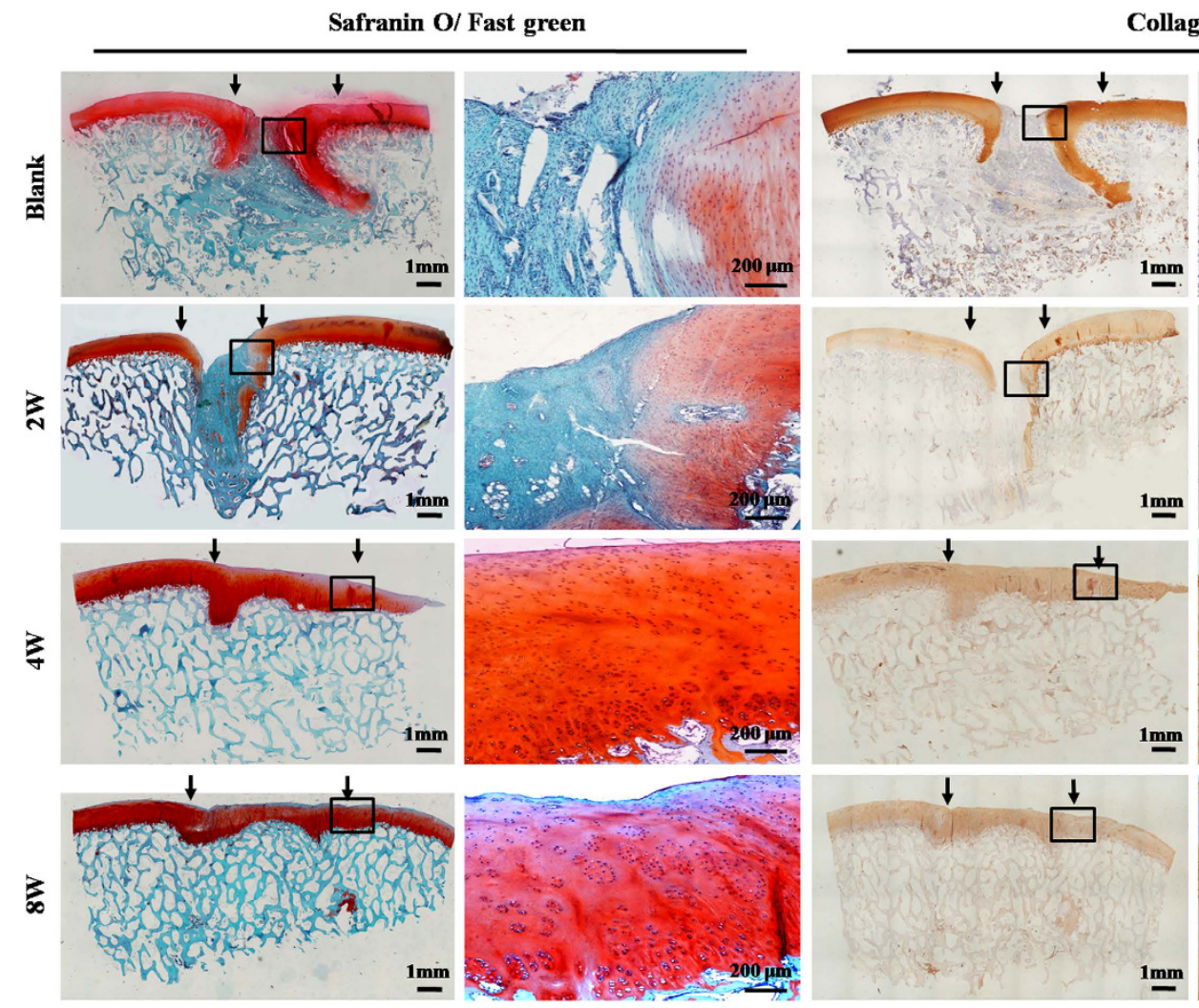

Collagen II
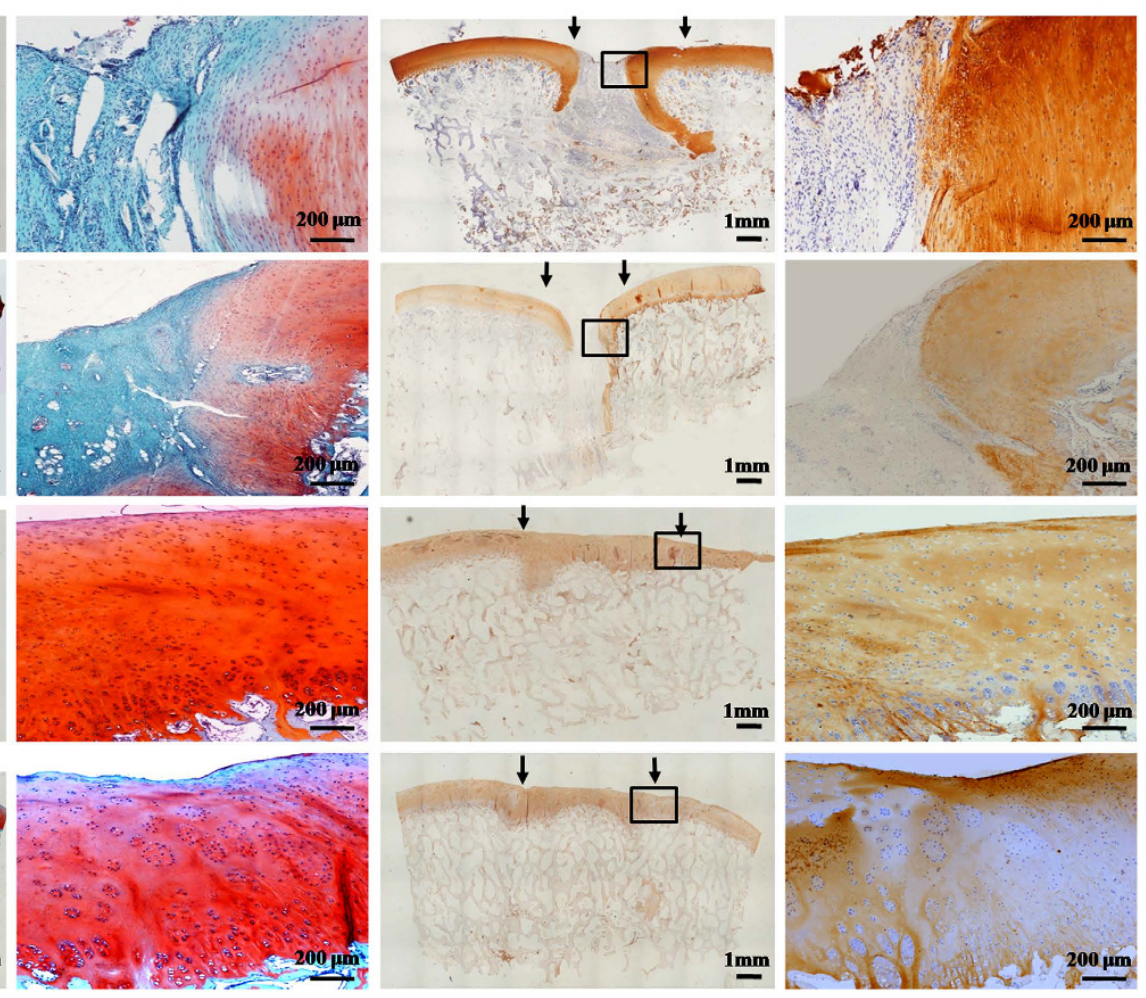

Figure 6. Cartilage-specific staining of repaired regions. Negative staining for Safranin-O and collagen type II was observed in the repaired tissue of the 2-week and blank groups. Repaired tissues in the cartilage defect regions of the 4- and 8-week groups showed continuous and strongly positive staining for Safranin-O and collagen II, but negative staining for these markers in bone defect regions. Black arrows indicate repaired regions.

expressions of hypertrophy-related genes provided a probability for bone regeneration of BEC-vitro through endochondral ossification pathways.

Despite this speculation, whether BEC-vitro could actually repair articular osteochondral defect remained uncertain. The current results revealed that BEC-vitro could successfully repair articular osteochondral defects in both the 4- and 8-week groups with ideal interface integration between repaired and native tissues. Most importantly, the implantation of BEC-vitro alone could realise tissue-specific repair of osteochondral defects with surface cartilage and underlying cancellous bone in these two groups. In contrast, neither cartilage nor subchondral bone regeneration was observed in the blank group, implying that implanted BEC-vitro might directly participate in cartilage and subchondral bone regeneration.

Why implantation of BEC-vitro alone could realise regeneration of both cartilage and subchondral bone is an important issue. It is widely accepted that BMSCs exhibit multiple lineage potentials, such that their ultimate in vivo fate is primarily determined by the implanted microenvironment ${ }^{25,26}$. Under articular cartilage microenvironment, endogenous growth factors in synovial fluid, mechanical stimulation, and hypoxia environment were favourable factors for driving chondrogenic differentiation of the implanted BMSCs ${ }^{27-29}$. Therefore, it is reasonable that BEC-vitro formed hyaline cartilage-like tissue under articular cartilage microenvironment in the current study. The most concerned issue is why BEC-vitro transformed into subchondral bone under bone defect environment. The following two reasons might account for this issue: 1) BEC-vitro had strong potential of endochondral ossification. Our previous study has demonstrated that BEC-vitro, especially within 8 weeks, had a high-risk of ectopic ossification after implanted into subcutaneous environment ${ }^{30}$. The high expressions of hypertrophy-related genes at both protein and gene levels in the current study further confirmed the endochondral ossification potential of BEC-vitro (Figs 2-3). (2) Regulation of osteogenic microenvironment. Once implanted into bone defect microenvironment, BEC-vitro might be stimulated by endogenous osteogenic factors, mechanical stimulation, and normoxia to transform into subchondral bone by activation of endochondral ossification pathways ${ }^{31,32}$. Although these factors provided reasonable explanations for the transform of BEC-vitro towards subchondral bone, the exact mechanisms still need to be further investigated.

Whether the maturity level of BEC-vitro affected the efficacy of osteochondral defect repair was another important issue. BMSC-scaffold constructs without adequate cartilage formation might only achieve limited cartilage regeneration because of a lack of sufficient in vitro chondrogenic induction and the influence of abundant un-degraded scaffolds ${ }^{13}$. However, fully mature engineered cartilage (similar to osteochondral arthroscopic grafting) might be difficult to integrate with surrounding native tissue and exhibit poor endochondral ossification 

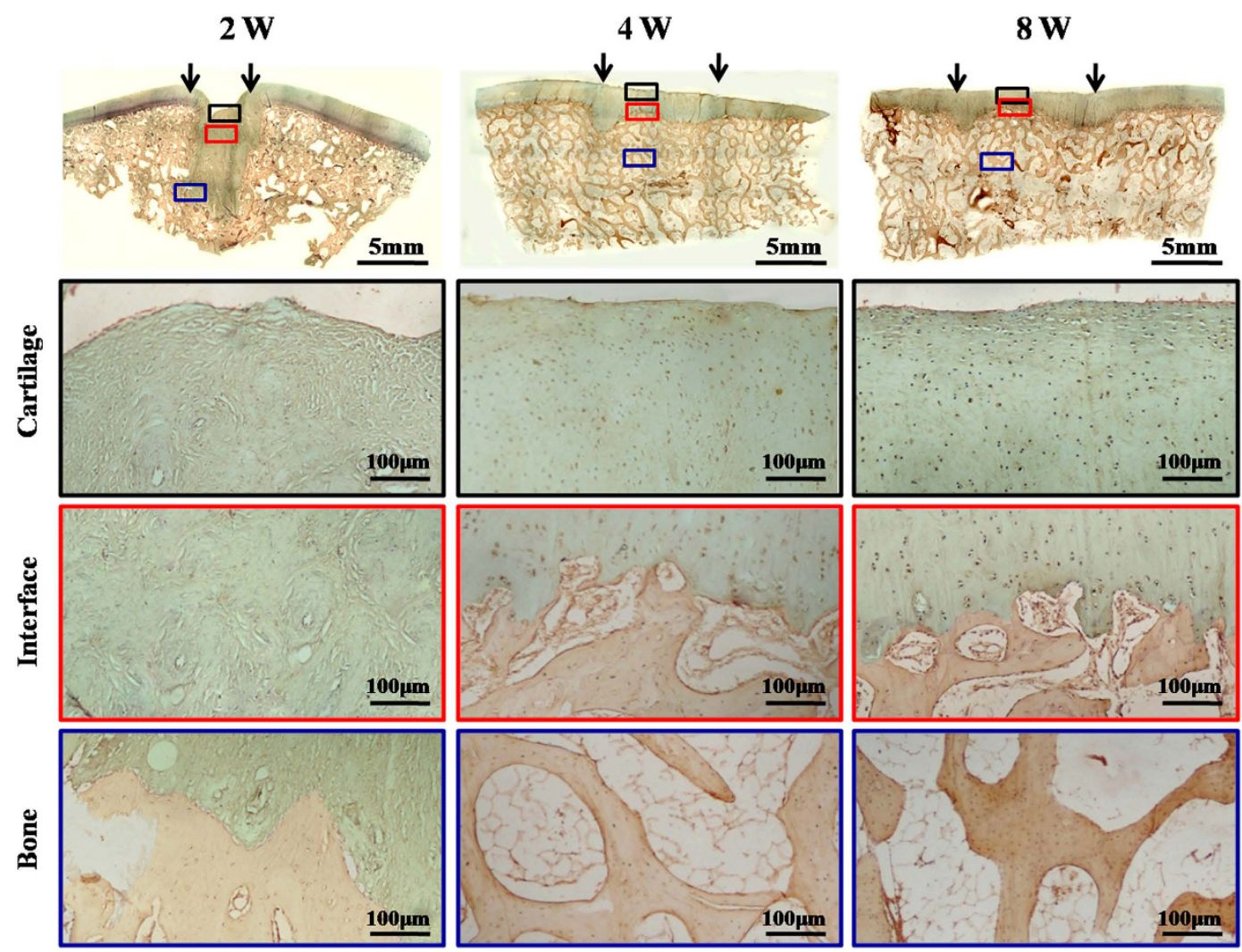

Figure 7. OCN immunohistochemical staining of repaired regions. In the 2-week group, the entire repaired region presented fibrous tissue with negative staining for OCN. In the 4- and 8-week groups, the repaired regions showed OCN-negative cartilaginous regions (black boxes) and OCN-positive subchondral bone regions (blue boxes) with distinct interfaces (red boxes), similar to the surrounding native structures. Black arrows indicate repaired regions.

potential $^{33,34}$. Therefore, determining an optimal time for in vivo implantation was the key in achieving satisfactory results for both osteochondral tissue regeneration and interface healing. The current results showed that satisfactory tissue regeneration and interface healing were only achieved in the 4 - and 8-week groups, but not in the 2-week group (mainly fibrous tissue formation). These results seemed to be inconsistent with the report of Miot S et al. ${ }^{35}$, which might be a result of different cell sources and scaffolds. For the current study, two key points might account for the failure observed in the 2-week group. First, BMSC-scaffold constructs at 2 weeks likely had difficulty developing into cartilage-like tissue after in vivo implantation because of a lack of sufficient chondrogenic factors and the negative influence of inflammatory cytokines in a surgical trauma niche ${ }^{24,27,29}$. Second, abundant remnant PGA fibres and their degradation products triggered serious inflammatory reactions, thus interrupting cartilage and bone regeneration in vivo ${ }^{16,17}$. In contrast, in the 4- and 8-week groups, BEC-vitro had basically formed cartilage-like tissues with less scaffold remnants, less dependence on extra growth factors, and stronger resistance to inflammatory cytokines in the trauma niche (protected by self-secreted ECM), and thus could proceed to develop into mature cartilage and subchondral bone in osteochondral microenvironments.

Although BEC-vitro presented successful repair results for articular osteochondral defects, the complete repair rate did not reach our expectations. Several factors might account for the unsatisfactory repair rate. First, large-size defects (10-mm diameter), which reached over $2 / 3$ of the femoral condyle area in the current study (Supplementary Fig. 4B), might significantly increase the difficulty of defect repair due to serious destruction of the local microenvironment and less mechanical support from surrounding cartilage ${ }^{36,37}$. Second, severe surgical trauma caused by too many defects (4 defects) in both legs might have negatively influenced osteochondral regeneration and articular function recovery ${ }^{37,38}$. Third, appropriate postoperative care and rehabilitation, which is difficult to guarantee in a large animal model, might impose another important negative factor ${ }^{39-41}$. Finally, individual differences among animals might also influence ultimate repair outcomes. Besides, for future clinical translation, other concerned issues such as biosafety, in vivo long-term fate, and the feasibility of repairing larger osteochondral defects, still need to be further investigated.

\section{Methods}

All procedures in the present study were strictly executed according to the regulations and laws of our country and in accordance with the Standing Committee on Ethics in China (The State Scientific and Technological Commission of China $)^{42}$. Animal experiments were approved by the Shanghai Jiao Tong University Committee on Animal Care and were conducted by the Key Laboratory of Tissue Engineering at Shanghai Ninth People's 

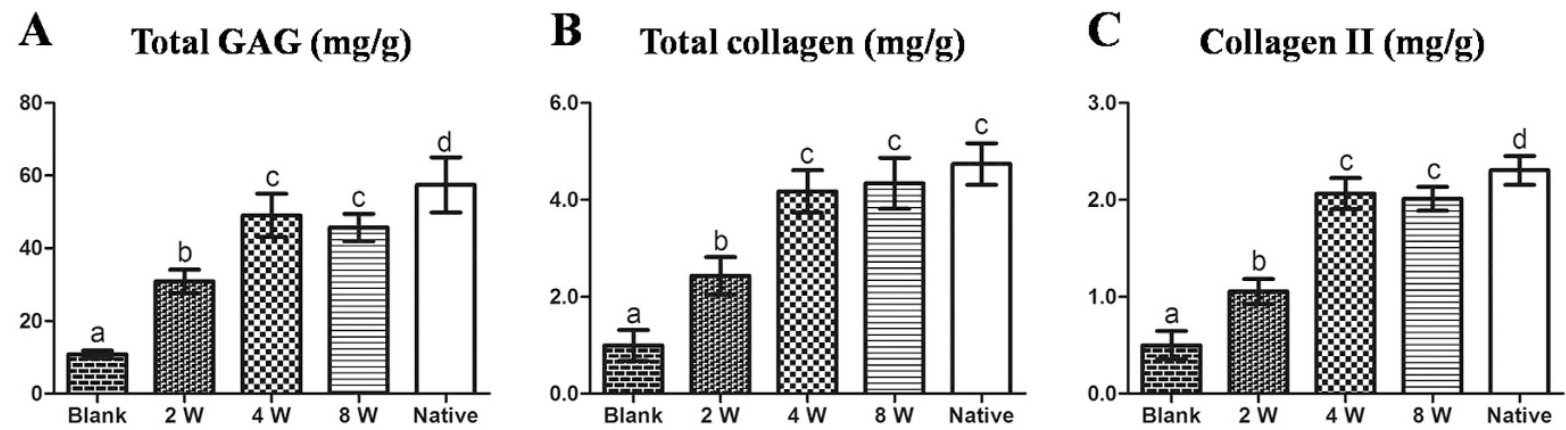

\section{Collagen I (mg/g)}
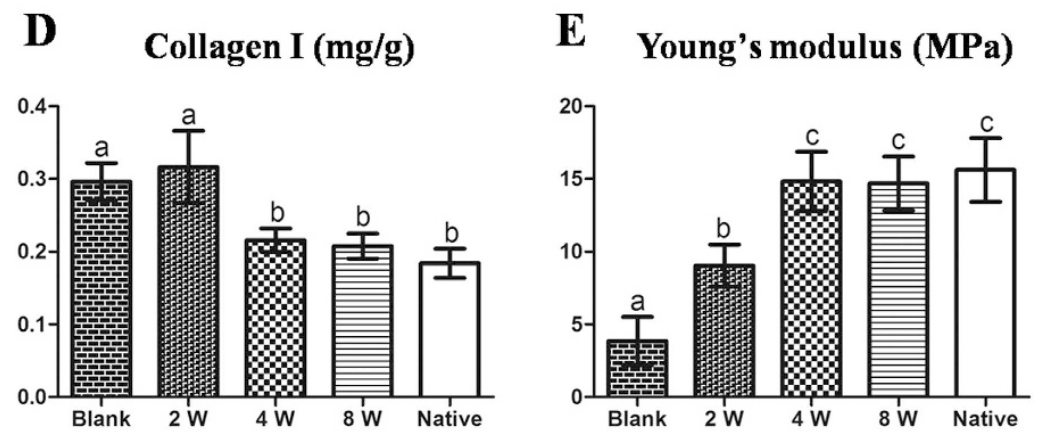

\section{F Comp . Loading(kgf)}

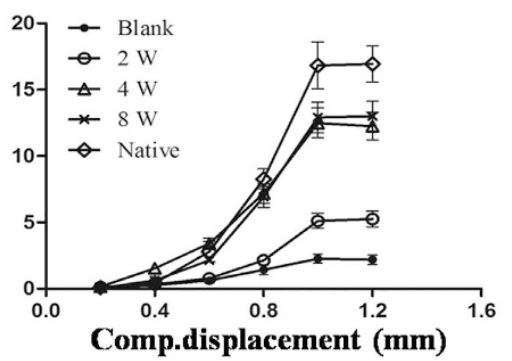

Figure 8. Biomechanical and biochemical evaluations of repaired tissue. Compared with the 2-week and blank groups, repaired tissues in the 4- and 8-week groups showed higher contents of GAG (A), total collagen (B) and collagen II (C), as well as increased Young's moduli (E) but lower collagen I content (close to native group levels) (D). Compressive loading-displacement curves showed different trends among groups (F). No significant differences were observed in the above examinations between the 4 - and 8 -week groups. Columns with different letters indicate statistical significance.

Hospital, affiliated with Shanghai Jiao Tong University in China. All experiments involving animals were performed in accordance with the approved guidelines.

General experimental design. A total of sixteen 6-month-old hybrid pigs ( 7 male and 9 female) weighing 45-50 kg were used in this research (Shanghai Jiagan Biological Technology Co., Shanghai, China). General experimental procedures included: isolation and expansion of BMSCs, in vitro cartilage engineering, evaluations of in vitro cartilage formation, establishment of osteochondral defect repair model, and evaluations of in vivo repair efficacy. BEC-vitro at 4 and 8 weeks was implanted in the experimental groups (4- and 8-week groups respectively), BEC-vitro at 2 weeks as cell-scaffold construct control, while untreated osteochondral defects served as a blank control.

Isolation and expansion of BMSCs. Bone marrow was aspirated from the anterior superior iliac spine of each animal. BMSCs were isolated, cultured and expanded in regular medium [Dulbecco's Modified Eagle's Medium (DMEM; Hyclone Laboratories, Logan, UT) containing 10\% foetal bovine serum (FBS, Hyclone Laboratories, Victoria, Australia), and 1\% Penicillin-streptomycin-amphotericin B (Hyclone Laboratories, Logan, UT) according to previously reported methods ${ }^{13,43}$. BMSCs were harvested at passage two for further experiments.

In vitro cartilage engineering using BMSCs. Fifteen milligrams of unwoven PGA fibres (National Tissue Engineering Center of China, Shanghai, China) were prepared into cylinders with a 10-mm diameter and 2-mm height, and then $0.5 \%$ PLA (Sigma-Aldrich, St. Louis, MO) solution was added to solidify the shape of PGA scaffolds ${ }^{30,44}$. Passage two BMSCs were harvested and seeded into PGA/PLA scaffolds at a concentration of $60 \times$ $10^{6}$ cells in $0.1 \mathrm{~mL} / \mathrm{scaffold}$, incubated for $4 \mathrm{~h}^{45}$, and then cultured in regular medium for $48 \mathrm{~h}$. After that, culture medium was replaced with chondrogenic medium [DMEM supplemented with $10 \mathrm{ng} / \mathrm{mL}$ transforming growth factor $\beta 1$ (TGF- $\beta 1$, HumanZyme, Chicago, IL), $40 \mathrm{ng} / \mathrm{mL}$ dexamethasone (Sigma-Aldrich), $100 \mathrm{ng} / \mathrm{mL}$ insulin-like growth factor 1 (IGF-1, R\&D Systems, Minneapolis, MN), and other addictives; Serum free], in which cells were cultured for 2-12 weeks.

SEM analysis of BMSC-scaffold constructs. The cell-scaffold constructs at various time points in vitro culture $(1,3,7$, and 14 days, $\mathrm{n}=3$ for each time point) and PGA/PLA scaffolds $(\mathrm{n}=3)$ were examined by SEM (Philips XL-30, Amsterdam, Netherlands) to evaluate attachment and ECM production of BMSCs on the scaffolds according to previously reported procedures ${ }^{46}$. 
Gross view and histological evaluation of BEC-vitro. At various time points from 2-10 weeks, BEC-vitro were grossly examined and immediately weighed. Next, samples were fixed $(n=3)$, embedded in paraffin, and cut into 5- $\mu$ m sections, which were then stained with haematoxylin and eosin (HE), Safranin-O (SO), COL II (monoclonal antibody ab34712, 1:100, Abcam, Cambridge, UK), COL I (monoclonal antibody 600-401104 S, 1:100, Rockland, PA) and COL X (monoclonal antibody ab49945, 1:100, Abcam, Cambridge, UK), according to our previously established methods ${ }^{47,48}$.

Biomechanical and biochemical evaluations of BEC-vitro. The compressive loading-displacement curve and Young's modulus of PGA-PLA scaffolds and BEC-vitro at 2, 4, and 8 weeks (conducted by static compression, biomechanical analyser, Instron-5542, Canton, MA, USA, $\mathrm{n}=5$ for each time point) were recorded and analysed according to a previously establish method ${ }^{44}$. After mechanical testing, samples were collected and minced for quantitative analysis of total $\mathrm{GAG}^{49}$, total collagen ${ }^{49}$, COL I, and COL II ${ }^{44,50}$. To determine the degradation rate of PGA scaffolds, BMSCs were digested from BMSC-scaffold constructs at various time points $(1,2$, $4,6,8,10$, and 12 weeks) with papain solution (1:50, Sigma-Aldrich). Next, remnant PGA fibres were collected, dried and weighed (PGA fibres were not digested by papain). Dry weight of remnant PGA fibres were recorded at each time point and plotted to form a PGA degradation curve $(n=3)$.

QRT-PCR analysis of BEC-vitro. RNA was extracted from engineered tissues at 2, 4, and 8 weeks, as well as from native cartilage $(n=5)$. A total of $2 \mu \mathrm{g}$ of RNA was used to reverse transcribe cDNA according to previously established methods ${ }^{51,52}$. QRT-PCR was performed according to the manufacturer's protocol (Thermo Fisher Scientific). Expression levels of COL IIA1, ACAN, SOX9, COL IA1, COL XA1, and MMP13 were analysed by qPCR using a LightCycler ${ }^{\circledR} 480$ system with a SYBR ${ }^{\circledR}$ green kit (Roche Molecular Biochemicals, Mannheim, Germany). Beta-actin housekeeping gene was used as an internal control. Forward and reverse primer pairs are listed in Supplementary Table 2.

Surgical procedures. As shown in Supplementary Fig. 4, two defects with a cylindrical shape (10-mm diameter, 4-mm depth) were created deep into subchondral bone at the weight-bearing area of the femoral medial and lateral condyles of the knee joint. After removal of blood clots, each defect was repaired randomly with either 2-, 4-, or 8-week constructs (two constructs from the same group were stacked together and implanted into the same defect), or left untreated. Constructs were fixed in place by stitching to surrounding native cartilage with biodegradable sutures. Most of the animals $(n=10)$ were created 4 defects in both knee joints for the implantation of both experimental groups and control groups, while other animals $(n=6)$ were only created 2 defects in one knee joint for the implantation of 4 - and 8-week BEC-vitro.

Gross observation and grading of the repaired regions. At 6 months post-operation, animals were euthanized to harvest repaired knee joints (distal part of femur). Samples were grossly examined to record surface smoothness, size of the repaired area, and healing interfaces with adjacent native cartilage. Next, samples were sawed sagittally at the midline to observe the healing interface between repaired and adjacent native osteochondral tissues. Gross view results were graded into three scales: complete repair, incomplete repair, and no repair, with criteria similar to a previous report ${ }^{53}$ (Detailed grading criteria were list in Supplementary Table 3).

Histological examination and grading of repaired regions. Harvested tissues were stained with $\mathrm{HE}$, Safranin-O/Fast green (SO/FG), COL II, COL I, and OCN (monoclonal antibody, 1:100, Abcam, Cambridge, UK) using the same procedure described above. Sirius red (SR) staining under polarized microscopy was also performed to evaluate the distribution of collagen type I according to a previously reported method ${ }^{54}$. To quantitatively evaluate cartilage regeneration, histological grading was performed by three persons through blind analysis, according to previously reported methods ${ }^{55,56}$ (Detailed histological grading scheme was list in Supplementary Table 4).

Biomechanical and biochemical analysis of the repaired tissues. The neocartilage in the reparative cartilage region of different groups $(n=5)$ were drilled and trimmed into a cylinder shape with a 4-mm diameter, and then used for biomechanical and biochemical analyses using the same procedures described above.

Statistical analysis. All quantitative data were recorded as mean \pm standard deviation. After confirmation of a normal data distribution, one-way analysis of variance and post-hoc least significant difference tests were used to determine statistical significance among the groups. A p-value less than 0.05 was considered statistically significant.

\section{Conclusion}

In summary, the current study demonstrated that i) in vitro cartilage formation and maturation using BMSCs represents a time-dependent manner; ii) implantation of BEC-vitro alone can realise tissue-specific repair of osteochondral defects with satisfactory interface integration; iii) the maturity level of BEC-vitro influences repair results. Although several factors that influence repair outcomes still require investigation, the current study provides a promising strategy for future clinical therapy of articular osteochondral defects.

\section{References}

1. Nukavarapu, S. P. \& Dorcemus, D. L. Osteochondral tissue engineering: current strategies and challenges. Biotechnol Adv 31, 706-721 (2013)

2. Kock, L., van Donkelaar, C. C. \& Ito, K. Tissue engineering of functional articular cartilage: the current status. Cell and Tissue Res 347, 613-627 (2012). 
3. Mobasheri, A., Csaki, C., Clutterbuck, A. L., Rahmanzadeh, M. \& Shakibaei, M. Mesenchymal stem cells in connective tissue engineering and regenerative medicine: applications in cartilage repair and osteoarthritis therapy. Histol Histopathol 24, 347-366 (2009).

4. Chow, J. C., Hantes, M. E., Houle, J. B. \& Zalavras, C. G. Arthroscopic autogenous osteochondral transplantation for treating knee cartilage defects: a 2- to 5-year follow-up study. Arthroscopy 20, 681-690 (2004).

5. Brittberg, M. et al. Treatment of deep cartilage defects in the knee with autologous chondrocyte transplantation. N Engl J Med 331, 889-895 (1994)

6. Komarek, J., Valis, P., Repko, M., Chaloupka, R. \& Krbec, M. [Treatment of deep cartilage defects of the knee with autologous chondrocyte transplantation: long-term results]. Acta Chir Orthop Traumatol Cech 77, 291-295 (2010).

7. Hunziker, E. B. Articular cartilage repair: basic science and clinical progress. A review of the current status and prospects. Osteoarthritis and Cartilage 10, 432-463 (2002).

8. Smith, G. D., Knutsen, G. \& Richardson, J. B. A clinical review of cartilage repair techniques. J Bone Joint Surg Br 87, 445-449 (2005).

9. Buckwalter, J. A. Articular cartilage injuries. Clin Orthop Relat Res 21-37 (2002).

10. Solchaga, L. A., Penick, K. J. \& Welter, J. F. Chondrogenic differentiation of bone marrow-derived mesenchymal stem cells: tips and tricks. Methods Mol Biol 698, 253-278 (2011).

11. Murdoch, A. D. et al. Chondrogenic differentiation of human bone marrow stem cells in transwell cultures: generation of scaffoldfree cartilage. Stem Cells 25, 2786-2796 (2007).

12. Gupta, P. K., Das, A. K., Chullikana, A. \& Majumdar, A. S. Mesenchymal stem cells for cartilage repair in osteoarthritis. Stem Cell Res Ther 3, 25 (2012).

13. Zhou, G. et al. Repair of porcine articular osteochondral defects in non-weightbearing areas with autologous bone marrow stromal cells. Tissue Eng 12, 3209-3221 (2006).

14. Filardo, G. et al. Mesenchymal stem cells for the treatment of cartilage lesions: from preclinical findings to clinical application in orthopaedics. Knee Surg Sports Traumatol Arthrosc 21, 1717-1729 (2013).

15. Tani, G., Usui, N., Kamiyama, M., Oue, T. \& Fukuzawa, M. In vitro construction of scaffold-free cylindrical cartilage using cell sheetbased tissue engineering. Pediatr Surg Int 26, 179-185 (2010).

16. Mauney, J. R. et al. Evaluation of gel spun silk-based biomaterials in a murine model of bladder augmentation. Biomaterials 32, 808-818 (2011)

17. Asawa, Y. et al. Early stage foreign body reaction against biodegradable polymer scaffolds affects tissue regeneration during the autologous transplantation of tissue-engineered cartilage in the canine model. Cell Transpl 21, 1431-1442 (2012).

18. Xuefeng, J., Yongcan, H., Yizhou, H., Chengguang, W. \& Li, D. [Expression profile of osteogenic-related genes during spontaneous calcification of rat bone marrow mesenchymal stem cells]. Zhongguo Xiu Fu Chong Jian Wai Ke Za Zhi 28, 133-141 (2014).

19. Lenas, P., Moos, M. \& Luyten, F. P. Developmental engineering: a new paradigm for the design and manufacturing of cell-based products. Part II: from genes to networks: tissue engineering from the viewpoint of systems biology and network science. Tissue Eng. Part B, Rev 15, 395-422 (2009).

20. Wood, J. J. et al. Autologous cultured chondrocytes: adverse events reported to the United States Food and Drug Administration. J Bone Joint Surg Am 88, 503-507 (2006).

21. Risbud, M. V. \& Sittinger, M. Tissue engineering: advances in in vitro cartilage generation. Trends Biotechnol 20, 351-356 (2002).

22. Jin, C. Z. et al. The maturity of tissue-engineered cartilage in vitro affects the repairability for osteochondral defect. Tissue Eng Part A 17, 3057-3065 (2011)

23. Getgood, A. et al. The Augmentation of a Collagen/Glycosaminoglycan Biphasic Osteochondral Scaffold with Platelet-Rich Plasma and Concentrated Bone Marrow Aspirate for Osteochondral Defect Repair in Sheep: A Pilot Study. Cartilage 3, 351-363 (2012).

24. Steinert, A. F. et al. Major biological obstacles for persistent cell-based regeneration of articular cartilage. Arthritis Res Ther 9, 213 (2007).

25. Pelttari, K. et al. Premature induction of hypertrophy during in vitro chondrogenesis of human mesenchymal stem cells correlates with calcification and vascular invasion after ectopic transplantation in SCID mice. Arthritis Rheum 54, 3254-3266 (2006).

26. De Bari, C., Dell'Accio, F. \& Luyten, F. P. Failure of in vitro-differentiated mesenchymal stem cells from the synovial membrane to form ectopic stable cartilage in vivo. Arthritis Rheum 50, 142-150 (2004).

27. Schmal, H. et al. In vivo quantification of intraarticular cytokines in knees during natural and surgically induced cartilage repair. Cytotherapy 11, 1065-1075 (2009).

28. Fortier, L. A., Mohammed, H. O., Lust, G. \& Nixon, A. J. Insulin-like growth factor-I enhances cell-based repair of articular cartilage. J Bone Joint Surg Br 84, 276-288 (2002).

29. Vasara, A. I., Konttinen, Y. T., Peterson, L., Lindahl, A. \& Kiviranta, I. Persisting high levels of synovial fluid markers after cartilage repair: a pilot study. Clin Orthop Relat Res 467, 267-272 (2009).

30. Liu, K. et al. The dependence of in vivo stable ectopic chondrogenesis by human mesenchymal stem cells on chondrogenic differentiation in vitro. Biomaterials 29, 2183-2192 (2008).

31. Scotti, C. et al. Recapitulation of endochondral bone formation using human adult mesenchymal stem cells as a paradigm for developmental engineering. Proc Natl Acad Sci USA 107, 7251-7256 (2010).

32. Dai, J. \& Rabie, A. B. VEGF: an essential mediator of both angiogenesis and endochondral ossification. J Dent Res 86, 937-950 (2007).

33. Borazjani, B. H. et al. Effect of impact on chondrocyte viability during insertion of human osteochondral grafts. J Bone Joint Surg Am 88, 1934-1943 (2006).

34. Marcacci, M. et al. Multiple osteochondral arthroscopic grafting (mosaicplasty) for cartilage defects of the knee: prospective study results at 2-year follow-up. Arthroscopy 21, 462-470 (2005)

35. Miot, S. et al. Influence of in vitro maturation of engineered cartilage on the outcome of osteochondral repair in a goat model. Eur Cell Mater 23, 222-236 (2012)

36. Emre, T. Y. et al. Factors affecting the outcome of osteochondral autografting (mosaicplasty) in articular cartilage defects of the knee joint: retrospective analysis of 152 cases. Arch Orthop Trauma Surg 133, 531-536 (2013).

37. Niemeyer, P., Salzmann, G. M., Hirschmuller, A. \& Sudkamp, N. P. Factors that influence clinical outcome following autologous chondrocyte implantation for cartilage defects of the knee. Z Orthop Unfall 150, 83-88 (2012).

38. Ebert, J. R. et al. Factors predictive of outcome 5 years after matrix-induced autologous chondrocyte implantation in the tibiofemoral joint. Am J Sports Med 41, 1245-1254 (2013).

39. Kreuz, P. C. et al. Importance of sports in cartilage regeneration after autologous chondrocyte implantation: a prospective study with a 3-year follow-up. Am J Sports Med 35, 1261-1268 (2007).

40. Viste, A. et al. Autologous chondrocyte implantation for traumatic full-thickness cartilage defects of the knee in 14 patients: 6-year functional outcomes. Orthop Traumatol Surg Res 98, 737-743 (2012).

41. Hambly, K., Bobic, V., Wondrasch, B., Van Assche, D. \& Marlovits, S. Autologous chondrocyte implantation postoperative care and rehabilitation: science and practice. Am J Sports Med 34, 1020-1038 (2006).

42. The State scientific and technological commission of China. Regulations for the Administration of Affaires Concerning Experimental Animals (Date of access: 01/08/2011) (2011)

43. Friedenstein, A. J., Chailakhyan, R. K. \& Gerasimov, U. V. Bone marrow osteogenic stem cells: in vitro cultivation and transplantation in diffusion chambers. Cell Tissue Kinet 20, 263-272 (1987). 
44. Yan, D. et al. The impact of low levels of collagen IX and pyridinoline on the mechanical properties of in vitro engineered cartilage. Biomaterials 30, 814-821 (2009).

45. Liu, X. et al. In vivo ectopic chondrogenesis of BMSCs directed by mature chondrocytes. Biomaterials 31, 9406-9414 (2010).

46. Cao, Y. et al. Bridging tendon defects using autologous tenocyte engineered tendon in a hen model. Plast Reconstr Surg 110, $1280-1289$ (2002).

47. Luo, X. et al. In vitro precultivation alleviates post-implantation inflammation and enhances development of tissue-engineered tubular cartilage. Biomed Mater 4, 025006 (2009).

48. Li, Q. et al. The role of bFGF in down-regulating alpha-SMA expression of chondrogenically induced BMSCs and preventing the shrinkage of BMSC engineered cartilage. Biomaterials 32, 4773-4781 (2011).

49. Spiller, K. L. et al. A novel method for the direct fabrication of growth factor-loaded microspheres within porous nondegradable hydrogels: controlled release for cartilage tissue engineering. J Control Release 157, 39-45 (2012).

50. Konishi, E., Shoda, M., Ajiro, N. \& Kondo, T. Development and evaluation of an enzyme-linked immunosorbent assay for quantifying antibodies to Japanese encephalitis virus nonstructural 1 protein to detect subclinical infections in vaccinated horses. $J$ Clin Microbiol 42, 5087-5093 (2004).

51. Kang, N. et al. Effects of co-culturing BMSCs and auricular chondrocytes on the elastic modulus and hypertrophy of tissue engineered cartilage. Biomaterials 33, 4535-4544 (2012).

52. Jiang, T. et al. Potent in vitro chondrogenesis of CD105 enriched human adipose-derived stem cells. Biomaterials 31, 3564-3571 (2010).

53. Sellers, R. S. et al. Repair of articular cartilage defects one year after treatment with recombinant human bone morphogenetic protein-2 (rhBMP-2). J Bone Joint Surg Am 82, 151-160 (2000).

54. Segnani, C. et al. Histochemical Detection of Collagen Fibers by Sirius Red/Fast Green Is More Sensitive than van Gieson or Sirius Red Alone in Normal and Inflamed Rat Colon. PloS one 10, e0144630 (2015).

55. Wakitani, S. et al. Mesenchymal cell-based repair of large, full-thickness defects of articular cartilage. J Bone Joint Surg Am 76, 579-592 (1994)

56. Pineda, S., Pollack, A., Stevenson, S., Goldberg, V. \& Caplan, A. A semiquantitative scale for histologic grading of articular cartilage repair. Acta Anat 143, 335-340 (1992)

\section{Acknowledgements}

This research was supported by Hi-Tech Research and Development Program of China (2012AA020507), National Natural Science Foundation of China (31271046, 81371703, 81371701, 81372091, and 81571823), Program for Shanghai Outstanding Medical Academic Leader, and Shanghai Rising-Star Program (16QB1400600), Shanghai Municipal Commission of Health and Family Planning (XBR2011050). The authors appreciate the technical supports and other helps from Demin Yin, Xiansong Wang, Lijuan Zong, Jinjun Chen, Wanyao Xia, Juanjuan Wu and Shuo Yin in the laboratory and National Tissue Engineering Center of China.

\section{Author Contributions}

H.A. and L.L. did all the primary technical undertaking, were responsible for the collection and analysis of data, and drafted the report. L.Y. developed the key techniques for in vitro engineered cartilage. L.X. and L.Y. assisted in the surgery procedures. L.F., Z.Z., Z.W., and L.W. participated in overall design and helped to refine the report. C.Y., Z.G. and W.X. were the principal investigators on the grant supporting the current work, designed the technical experiments, participated in the surgical procedures and oversaw the collection of results, and data interpretation.

\section{Additional Information}

Supplementary information accompanies this paper at http://www.nature.com/srep

Competing financial interests: The authors declare no competing financial interests.

How to cite this article: He, A. et al. Repair of osteochondral defects with in vitro engineered cartilage based on autologous bone marrow stromal cells in a swine model. Sci. Rep. 7, 40489; doi: 10.1038/srep40489 (2017).

Publisher's note: Springer Nature remains neutral with regard to jurisdictional claims in published maps and institutional affiliations.

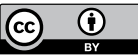

This work is licensed under a Creative Commons Attribution 4.0 International License. The images or other third party material in this article are included in the article's Creative Commons license, unless indicated otherwise in the credit line; if the material is not included under the Creative Commons license, users will need to obtain permission from the license holder to reproduce the material. To view a copy of this license, visit http://creativecommons.org/licenses/by/4.0/

(c) The Author(s) 2017 